\title{
Response: Commentary: Decomposition of Heart Rate Variability Spectrum into a Power-Law Function and a Residual Spectrum
}

\author{
Jane Kuo ${ }^{1}$ and Cheng-Deng Kuo ${ }^{2,3 *}$ \\ ${ }^{1}$ Department of Dentistry, Cathay General Hospital, Taipei, Taiwan, ${ }^{2}$ Division of Chest Medicine, Department of Internal \\ Medicine, Changhua Christian Hospital, Changhua, Taiwan, ${ }^{3}$ Department of Medical Research, Taipei Veterans General \\ Hospital, Taipei, Taiwan
}

Keywords: decomposition, heart rate variability, power-law function, residual spectrum, power spectral analysis, fractal

\section{A Commentary on}

OPEN ACCESS

Edited by:

Shimon Rosenheck,

Meir Medical Center, Israel

Reviewed by:

Yael Yaniv,

Technion Israel Institute of

Technology, Israel

*Correspondence:

Cheng-Deng Kuo

cdkuo23@gmail.com

Specialty section:

This article was submitted to

Cardiac Rhythmology,

a section of the journal

Frontiers in Cardiovascular Medicine

Received: 31 July 2018 Accepted: 17 December 2018 Published: 17 January 2019

Citation:

Kuo J and Kuo C-D (2019) Response: Commentary: Decomposition of Heart Rate Variability Spectrum into a

Power-Law Function and a Residual Spectrum. Front. Cardiovasc. Med. 5:191 doi: 10.3389/fcvm.2018.00191
Commentary: Decomposition of Heart Rate Variability Spectrum into a Power-Law Function and a Residual Spectrum

by Castiglioni, P. (2018). Front. Cardiovasc. Med. 5:94. doi: 10.3389/fcvm.2018.00094

Dr. Paolo Castiglioni (1) comments on our paper "Decomposition of heart rate variability spectrum into a power-law function and a residual spectrum" (2). In that commentary, he pointed out that both the $\operatorname{rg}(=s \cdot \log (F r q)+Y)$ in Equation (1) in his commentary and the PSDrg have the units of PSD, or $\mathrm{ms}^{2} / \mathrm{Hz}$, so that the spectral ratio rPSD in Equation (3) in his commentary is a dimensionless number. In the above argument, the "rg" denotes "regression," "PSD" denotes "power spectral density," and "rPSD” denotes "residual PSD." We are grateful to Dr. Castiglioni for his careful examination of our equations. Unfortunately, the argument of Dr. Catiglioni was based on the misunderstanding caused by the omission of the units of the variables in our Equation (2). To avoid confusion and misunderstanding, we shall re-express our equations in the followings with the units of the variables placed in suitable positions in the equations.

To facilitate the decomposition of the PSD in the whole heart rate variability (HRV) spectrum, the power-law relation of HRV can be obtained by plotting $\log \left(\frac{P S D}{\mathrm{~ms}^{2} / \mathrm{Hz}}\right)$ against $\log \left(\frac{F r q}{H z}\right)$ within the frequency range from $>0 \mathrm{~Hz}$ to the Nyquist frequency. Both $\frac{P S D}{\mathrm{~ms}^{2} / \mathrm{Hz}}$ and $\frac{F r q}{H z}$ are dimensionless. The $0 \mathrm{~Hz}$ point must be excluded because $\log (0)$ is not defined mathematically. The linear regression relation between $\log \left(\frac{\mathrm{PSD}}{m s^{2} / \mathrm{Hz}}\right)$ and $\log \left(\frac{F r q}{H z}\right)$ in the PSD of HRV can be expressed as

$$
\log \left(\frac{P S D_{r g}}{\mathrm{~ms}^{2} / \mathrm{Hz}}\right)=s \cdot \log \left(\frac{F r q}{H z}\right)+Y
$$

where the "log" denotes logarithm, the subscript "rg" denotes "regression," and the " $s$ " and "Y" are the "slope" and "Y-intercept" of linear regression between $\log \left(\frac{\mathrm{PSD}_{\mathrm{rg}}}{\mathrm{ms}^{2} / \mathrm{Hz}}\right)$ and $\log \left(\frac{F r q}{H z}\right)$, respectively. 
Equation (1) can be re-written as

$$
\frac{\mathrm{PSD}_{\mathrm{rg}}}{\mathrm{ms}^{2} / \mathrm{Hz}}=10^{s \cdot \log \left(\frac{\mathrm{Frq}}{\mathrm{Hz}}\right)+Y}=10^{Y} \cdot\left(\frac{\mathrm{Frq}}{\mathrm{Hz}}\right)^{s} .
$$

Equation (2) shows that the $\frac{\mathrm{PSD}_{\mathrm{rg}}}{\mathrm{ms}^{2} / \mathrm{Hz}}$ is a power-law function of $\left(\frac{\mathrm{Frq}}{\mathrm{Hz}}\right)$. The difference between $\log \left(\frac{\mathrm{PSD}}{\mathrm{ms}^{2} / \mathrm{Hz}}\right)$ and $\log \left(\frac{\mathrm{PSD}_{\mathrm{rg}}}{\mathrm{ms}^{2} / \mathrm{Hz}}\right)$ is the logarithm of the residual power spectral density, $\frac{\mathrm{rPSD}}{\mathrm{ms}^{2} / \mathrm{Hz}}$, that cannot be accounted for by the $\frac{\mathrm{PSD}_{\mathrm{rg}}}{\mathrm{ms}^{2} / \mathrm{Hz}}$ :

$$
\log \left(\frac{\mathrm{rPSD}}{\mathrm{ms}^{2} / \mathrm{Hz}}\right)=\log \left(\frac{\mathrm{PSD}}{\mathrm{ms}^{2} / \mathrm{Hz}}\right)-\log \left(\frac{\mathrm{PSD}_{\mathrm{rg}}}{\mathrm{ms}^{2} / \mathrm{Hz}}\right)=\log \left(\frac{\mathrm{PSD}}{\mathrm{PSD}_{\mathrm{rg}}}\right)
$$

Equations $(2,3)$ give immediately the following expression of rPSD:

$$
\mathrm{rPSD}=\frac{\mathrm{PSD}}{\mathrm{PSD}_{\mathrm{rg}}} \cdot\left(\mathrm{ms}^{2} / \mathrm{Hz}\right)=\mathrm{PSD} \cdot 10^{-\mathrm{Y}} \cdot\left(\frac{\mathrm{Frq}}{\mathrm{Hz}}\right)^{-\mathrm{s}} .
$$

Since the PSDrg has the unit of $\mathrm{ms}^{2} / \mathrm{Hz}$, the rPSD also has the same unit of $\mathrm{ms}^{2} / \mathrm{Hz}$ as that of PSD, according to Equation (4). Thus, the rPSD is not dimensionless, as asserted by Dr. Castiglioni (1). Because the rPSD is obtained from the original PSD by removing the power law constituent in it, its frequency components are all significantly smaller than those of the original PSD. This is comprehensible.

Castiglioni also commented on our fitting of the regression line up to the Nyquist frequency (1). He asserted that the PSDrg cannot be considered the true "fractal component" of the spectrum, because the regression slope $s$ is influenced by the oscillations in the low-frequency (LF) and high-frequency (HF) bands. Though the PSDrg can be influenced by the oscillations in the LF and HF bands, its overall behavior is self-similar with respect to frequency because the PSDrg is a power-law function of frequency, according to Equation (2). It is doubtful that the PSDrg cannot be interpreted as a fractal. If the PSDrg cannot be interpreted as a "fractal," then call it a power-law function of frequency. It is not important whether the power-law function

\section{REFERENCES}

1. Castiglioni P. Commentary: decomposition of heart rate variability spectrum into a power-law function and a residual spectrum. Front Cardiovasc Med. (2018) 5:94. doi: 10.3389/fcvm.2018.00094

2. Kuo J, Kuo CD. Decomposition of heart rate variability spectrum into a powerlaw function and a residual spectrum. Front Cardiovasc Med. (2016) 3:16. doi: $10.3389 /$ fcvm.2016.00016

3. Kobayashi M, Musha T. 1/f fluctuation of heartbeat period. IEEE Trans Biomed Eng. (1982) 29:456-7.

4. Otsuka K, Nakajima S, Yamanaka T. Vagal tone and its association with a new index of heart rate variability called 1/f fluctuations. J Ambul Monit. (1994) $7: 213-18$.

5. Task Force of the European Society of Cardiology and the North American Society of Pacing and Electrophysiology. Heart rate variability. Standards of measurement, physiological interpretation, and clinical use. Eur Heart J. (1996) 17:354-81.

6. Sassi R, Cerutti S, Lombardi F, Malik M, Huikuri HV, Peng CK, et al. Advances in heart rate variability signal analysis: joint position
PSDrg obtained in our decomposition method can be regarded as a fractal or not.

The purpose of decomposing the PSD of HRV into a powerlaw function of frequency and a residual PSD is to examine more closely the heart rate oscillations in the LF and HF regions, rather than to examine the frequency region below $0.04 \mathrm{~Hz}$, because LF and HF regions are mostly concerned in many clinical settings that are associated with autonomic dysfunction of the patients. If we adhere to the international guidelines on HRV to analyze the PSD within the frequency range of $<0.04 \mathrm{~Hz}(3-7)$, then the heart rate oscillations in the LF and HF regions cannot be examined in more details by using the decomposition techniques introduced by us in our previous article (2).

The PSD of HRV obtained from either short-term or longterm $(6,7)$ recordings of heart periods can be decomposed into a power-law function and a residual spectrum by using the mathematical technique devised by our group. The point is that if we are going to examine the LF and HF components of HRV in more details by using the decomposition method, then fitting the regression line between $\log \left(\frac{\mathrm{PSD}}{\mathrm{ms}^{2} / \mathrm{Hz}}\right)$ and $\log \left(\frac{\mathrm{Frq}}{\mathrm{Hz}}\right)$ beyond the upper frequency limit $(0.04 \mathrm{~Hz})$ of the very low frequency (VLF) component is a necessity. Otherwise, the PSD within the frequency range of LF and HF will not be decomposed, and the LF and HF components of HRV cannot be examined in more details by using the decomposition method developed by our group. Thus, the decomposition of the PSD of HRV within the whole frequency range from $>0$ to the Nyquist frequency is the simplest way of performing the decomposition analysis.

\section{AUTHOR CONTRIBUTIONS}

JK: drafting of the manuscript. C-DK: conception, design and finalization of the work.

\section{FUNDING}

This work was supported by the grant 106-CCH-IRP-100 from the Changhua Christian Hospital, Changhua, Taiwan.

statement by the e-Cardiology ESC Working Group and the European Heart Rhythm Association co-endorsed by the Asia Pacific Heart Rhythm Society. Europace (2015) 17:1341-53. doi: 10.1093/europace/e uv015

7. Kazuma N, Nozaki M, Nakamura E, Matsuoka I, Otani T. Biological rhythm in $1 / \mathrm{f}$ fluctuations of heart rate in asthmatic children. Allergol Intern (2004) 53:265-9. doi: 10.1111/j.1440-1592.2004.0 0343.x

Conflict of Interest Statement: The authors declare that the research was conducted in the absence of any commercial or financial relationships that could be construed as a potential conflict of interest.

Copyright (C) 2019 Kuo and Kuo. This is an open-access article distributed under the terms of the Creative Commons Attribution License (CC BY). The use, distribution or reproduction in other forums is permitted, provided the original author(s) and the copyright owner(s) are credited and that the original publication in this journal is cited, in accordance with accepted academic practice. No use, distribution or reproduction is permitted which does not comply with these terms. 